\title{
Field evaluation of effectiveness of the BG-Sentinel, a new trap for capturing adult Aedes aegypti (Diptera: Culicidae)
}

\author{
Rafael Maciel-de-Freitas, Álvaro Eduardo Eras*, Ricardo Lourenço-de-Oliveira/ ${ }^{+}$
}

\begin{abstract}
Laboratório de Transmissores de Hematozoários, Instituto Oswaldo Cruz-Fiocruz, Av. Brasil 4365, 21040-900 Rio de Janeiro, RJ, Brasil *Laboratório de Culicídeos, Departamento de Parasitologia, Instituto de Ciências Biológicas, Universidade Federal de Minas Gerais, Belo Horizonte, MG, Brasil
\end{abstract}

\begin{abstract}
In recent years, the development of new tools to gather field information about vector ecological parameters has increased. This report evaluated the BG-Sentinel Trap (BGS-Trap), a promising new attempt to improve collection of the dengue vector, Aedes aegypti. The efficacy of the BGS-Trap was compared with the CDC backpack aspirator, one of the commonest used methods for capturing adult mosquitoes. BGS-Traps captured significantly more Ae. aegypti males $\left(\chi^{2}=21.774, d f=1, P<0.05\right)$ and females $\left(\chi^{2}=56.007, d f=1, P<0.05\right)$ than CDC aspirator during all days of field collection. However, CDC aspirator was significantly more efficient to capture Culex quinquefasciatus males $\left(\chi^{2}=5.681, d f=1, P<0.05\right)$ and females $\left(\chi^{2}=6.553, d f=1, P<0.05\right)$. BGS-Traps captured host-seeking females (varying between 68.75 to $89.8 \%$ ) in detriment of females in other behavioral and physiological stages. BGS-Traps proved to be efficient and can be used for monitoring adult mosquito populations.
\end{abstract}

Key words: Stegomyia - Culex quinquefasciatus - trap - gonotrophic cycle - dengue - yellow fever

Sampling the adult mosquito population can provide important information on vector ecology and behavior (Kröckel et al. 2006). Field collected data can increase the efficiency of vector control and consequently decrease dengue transmission (Focks 2003). Several methods and traps have been developed to maximize the number of adults captured (for an extensive list of examples see Service 1993). However, the most used are sticky ovitraps, visual traps, and backpack aspirators (Clark et al. 1994, Jensen et al. 1994, Ordonez-Gonzalez et al. 2001). Obviously, each trap methodology has its own disadvantages (Service 1993). For instance, sticky ovitraps can damage sampled mosquitoes, backpack aspirator results may vary with skills and motivation of inspectors, and visual traps can be of lower efficiency in comparison with other collection methods (Service 1993).

The development of an efficient trapping method to collect dengue and yellow fever mosquito Aedes aegypti that can give a quick, realistic, and standardized estimation of mosquito biological parameters, such as adult population density, survival, and virus circulation is a key for dengue control campaigns. Recently, a new mosquito trap has been used to collect field data from Ae. aegypti, the BG-Sentinel Trap ${ }^{\mathrm{TM}}$. The BG-Sentinel Trap (BGS-Trap) uses a blend of mosquito attractants consisting of lactic acid, ammonia, and caproic acid, substances which are

Financial support: Unesco (grant FC23467/2004), CNPq (grant 501742/2003-4), Faperj, Ministério da Saúde (SCT.E/DECIT) ${ }^{+}$Corresponding author: lourenco@ioc.fiocruz.br

Received 6 February 2006

Accepted 24 April 2006 also found on human skin. The blend is constantly emitted in a fixed ratio from a long lasting multi-component dispenser, the BG-Lure (Kröckel et al. 2006).

The aims of this report were to evaluate (1) the efficiency of the BGS-Trap by comparison with the most used method for alive adult mosquito sampling, the CDC backpack aspirator (John W Hock Company), which has been frequently used to collect mosquitoes in a neighborhood in Rio de Janeiro (Maciel-de-Freitas et al. 2006, LimaCamara et al. 2006); (2) the preferential location (inside houses or in the peridomestic area) where BGS-Traps should be placed; and (3) the ovarian physiological stage of Ae. aegypti females captured with the BGS-Trap

\section{MATERIALS AND METHODS}

Study area - The study was conducted in an essentially remote district of Rio de Janeiro city, named Tubiacanga ( $\left.22^{\circ} 47^{\prime} 08^{\prime \prime} \mathrm{S} 43^{\circ} 13^{\prime} 36^{\prime \prime} \mathrm{W}\right)$, which is located in Governador's Island, a lowland coastal area, partially surrounded by the Guanabara Bay shores and a three-meter wall of the Tom Jobim International Airport of Rio de Janeiro and its numerous landing stripes. Thus, mosquito emigration is not expected to happen in large scale. Tubiacanga has approximately 2900 inhabitants living in around 870 houses arranged in 14 blocks distributed through 8.6 hectare, and its nearest neighborhood is Parque Royal, located $2.1 \mathrm{~km}$ apart. Houses usually have two bedrooms, with large and shaded peridomestic areas.

BGS-Trap - The BGS-Trap has been recently developed by BioGents GmbH (Regensburg, Germany) and utilizes patent pending technology from the University of Regensburg. The trap consists of an easy to transport, collapsible white bucket with white gauze covering its opening. In the middle of the gauze cover, there is a black tube through which a down flow is created by $12 \mathrm{~V}$ DC fan that causes any mosquito in the vicinity of the opening to be sucked into a catch bag. The catch bag is located be- 
fore the suction fan, therefore avoiding damage to specimens passing through the fan. The air then exits the trap through the large surface of white gauze $\left(987 \mathrm{~cm}^{2}\right)$ : the design therefore generates ascending currents (Fig. 1). These are similar to convection currents produced by a human host, both in its direction, its geometrical structure, and, due to the addition of attractants, also in its composition. The attractants are given off by the BG-Lure (BioGents $\mathrm{GmbH}$, Germany), a dispenser which releases a defined combination of lactic acid, ammonia, and caproic acid, substances that are found on human skin. The dispenser emits the attractants for up to five months. During the tests, the BGS-Traps were simply placed on the ground.

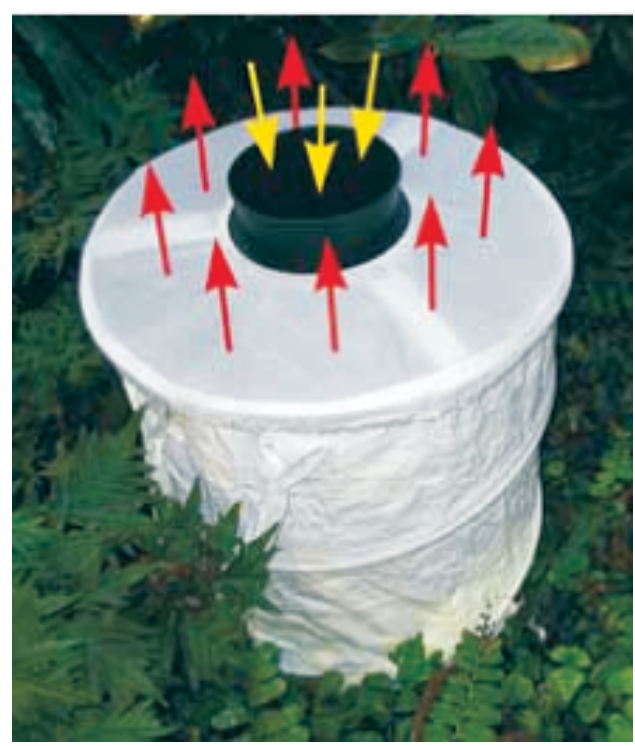

Fig. 1: the BG Sentinel-Trap. Mosquitoes flying in trap vicinity are attracted by a dispenser which releases a combination of components found in human skin and are captured in the black centered catch bag (yellow arrows). The air exits the trap through the large surface of white gauze (red arrows), generating ascending currents

Comparison between BGS-Trap and backpack aspiration - Thirty houses of Tubiacanga were daily randomly selected: fifteen houses for BGS-Trap placement and fifteen houses for backpack aspiration. BGS-Traps were installed indoors (inside house) and outdoors (in the peridomestic area), for posterior comparisons regarding the place where it is more efficient. BGS-Traps operated for $24 \mathrm{~h}$ a day. Aspirations were carried out for about 20 min indoors and outdoors. Captures were carried out daily for a period of ten days, and captured mosquitoes were taken to laboratory, where species identification was performed.

Mosquitoes - Ae aegypti used in the mark-releaserecapture experiments were derived from a laboratory colony that is constantly renewed with eggs collected in Rio de Janeiro city with oviposition traps. Larvae were fed with fish food (Tetramin $\left.{ }^{\circledR}\right)$ and reared according to Consoli and Lourenço-de-Oliveira (1994). After emergence, females and males were kept together at $25 \pm 3^{\circ} \mathrm{C}$ and $65 \pm$ $5 \% \mathrm{RH}$ and provided with sucrose solution. A meal with human blood was offered around $8 \mathrm{~h}$ before releasing for two groups of females (Cohorts 1 and 2), as explained below. Blood meals were offered in an apparatus previously described by Rutledge (1964).

Mosquito cohorts - Mosquito rearing was planned to obtain three ages and/or physiological cohorts to simultaneous releasing: (1) old-aged parous females (released after 11-12 days after emergence) and blood-engorged, (2) new-aged nulliparous females (released with 5-6 days after emergence) and blood-engorged, and (3) new-aged non blood-fed nulliparous females. Females belonging to Cohort 1 have also taken a previous blood-meal on day 5 after emergence and fully engorged females were kept for the following 6-days in a cage provided with cups with water for oviposition. A second blood meal was offered to these females around $8 \mathrm{~h}$ before releasing and only those fully engorged were dust marked and released.

By releasing females in three distinct physiological stages, females with different host-seeking behaviors were expected to be produced, and the number of females captured by the BGS-Trap would vary as a consequence of these differences. For example, females from Cohort 3 , the unique without a blood meal, should seek for a host immediately after release, while blood-fed females from Cohorts 1 and 2 would be host-seeking after blood digestion. Consequently, a high number of captured females from Cohort 3 immediately after release was expected. We also expected to capture high numbers of females from Cohorts 1 and 2 on days two or three after release.

Marking, releasing and capturing (MRC) - Released mosquitoes were marked with different colors of fluorescent dust (Day-Glo Color Corp., Cleveland, Ohio) in small cylindrical cages $(12 \times 10 \mathrm{~cm})$ and released in approximately $1 \mathrm{~h}$ after dust marking. The 458,412 , and $319 \mathrm{fe}-$ males belonging to Cohorts 1, 2, and 3 were marked with pink, yellow, and orange dust, respectively. Marked mosquitoes were released in the afternoon (at 5-6 pm). Thirty females belonging to Cohort 1 were randomly selected before releasing and immediately dissected for determination of parity rate according to Detinova $(1945,1949)$. Essentially all (97\%) of dissected females belonging to Cohort 1 have already passed a gonotrophic cycle before releasing, thus considered parous.

In the MRC experiments, dust marked females were daily collected only with BGS-Traps. Collection period lasted only four days, once the physiological stage of different cohorts would start to overlap from this day on. Captured mosquitoes were carried to laboratory, and examined under UV light, separated according to dust color, counted and identified according to species. All collected Ae. aegypti were dissected for the determination of ovarian development stage according to Christophers (1911). For classification purposes, ovarian developmental stages I, I-II, and II were grouped together as initial stages of development. Stages III and IV were grouped as intermediary stages and ovarian in stage $\mathrm{V}$ was classified as final stage, once females would be gravid (Tsuda et al. 2001). 
Data analysis - Comparisons between the total number of mosquitoes caught in the BGS-Traps and backpack aspirators were carried out by a chi-square test. The efficiency of the BGS-Trap was observed not only to the total number of mosquitoes collected, but also for each day of collection. For that, the number of mosquitoes of a single gender and species captured in BGS-Traps was subtracted by the number of mosquitoes of a single gender and species captured in the backpack aspirator. When a positive result was obtained, BGS-Trap was considered to be more efficient. Meanwhile, when a negative result was achieved, backpack aspirator was considered to be more efficient. This approach leads us to discriminate if one collecting method was more efficient only in the total number gathered or in various days of field work.

The number of mosquitoes collected indoors and outdoors by BGS-Traps was non-normal for all species or gender tested, according to the Kolmogorov-Smirnov normality test. Thus, comparisons concerning the best location for installing the BGS-Trap were performed using the Mann-Whitney test (Sokal \& Rohlf 2001).

Ethical considerations - Mark-release-capture experimental protocols were submitted to and approved by Fiocruz Ethical Committee (CEP/Fiocruz protocol number P0079-99).

\section{RESULTS}

BGS-Trap efficiency - Only two mosquito species were captured by both BGS-Trap and backpack aspirators: Ae. aegypti and Culex quinquefasciatus (Table I). Comparing the total number of mosquitoes captured, by species and gender, the backpack aspirator was statistically more efficient to capture $C x$. quinquefasciatus males $\left(\chi^{2}=5.681\right.$, $\mathrm{df}=1, \mathrm{P}<0.05)$ and females $\left(\chi^{2}=6.553, \mathrm{df}=1, \mathrm{P}<0.05\right)$. However, the BGS-Trap was extremely more efficient to capture Ae. aegypti males $\left(\chi^{2}=21.774, \mathrm{df}=1, \mathrm{P}<0.05\right)$ and females $\left(\chi^{2}=56.007, \mathrm{df}=1, \mathrm{P}<0.05\right)$.

BGS-Trap was more efficient than backpack aspirator to collect Ae. aegypti females during all days of field experiments (Fig. 2). Similar results were obtained for Ae. aegypti males; BGS-Trap was less efficient than backpack aspirator in a unique day. However, different collection patterns were observed for $C x$. quinquefasciatus. The BGS-Trap was less effective than the backpack aspirator in four and five days for $C x$. quinque-fasciatus male and female, respectively. These results suggest higher efficiency and specificity of BGS-Trap to capture both genders of adult Ae. aegypti than $C x$. quinquefasciatus.

BGS-Traps installed in the outdoor area captured significantly more $C x$. quinquefasciatus males $(\mathrm{U}=499.0, \mathrm{P}=$ $0.0093)$. However, similar numbers of captured $C x$. quinquefasciatus females $(\mathrm{U}=683.0, \mathrm{P}=0.3524), A e$. aegypti males $(\mathrm{U}=157.5, \mathrm{P}=0.7587)$, and Ae. aegypti females $(\mathrm{U}=229.0, \mathrm{P}=0.2925)$ were observed regardless whether the BGS-Trap was installed inside houses or in the peridomestic area.

In the MRC experiments, the number of recovered dust marked females was similar with the expected pattern. Therefore, a high number of females from Cohort 1 and 2 were captured after two days post-release and females from Cohort 3 were captured in a high number immediately after release (Table II). Cohorts with parous and nulliparous females had higher recapture rates, varying from 6.98 to $15.36 \%$ for Cohorts 1 and 3, respectively.

Results from each cohort are presented in Fig. 3. Notably, females with ovarian development in stages I, I-II, and II, i.e. host-seeking females, were captured in higher frequency in all cohorts. In Cohort 1, 22 (68.7\%) females had ovarioles in initial stages, $8(25 \%)$ had intermediary stages, and just $2(6.25 \%)$ were gravid. In Cohort 2, 25 (75.7\%) females had initial ovarian developmental stages, $6(18.2 \%)$ in intermediary stages, and $2(6 \%)$ were gravid. Remarkably, during the first two days of capture only host-

TABLE II

Number of Aedes aegypti females with different ovarian developmental stages captured in BG-Sentinels during a markrelease-recapture study

\begin{tabular}{lccc}
\hline Days after release & Cohort 1 & Cohort 2 & Cohort 3 \\
\hline 1 & 3 & 1 & 14 \\
2 & 6 & 5 & 21 \\
3 & 14 & 15 & 6 \\
4 & 9 & 12 & 8 \\
\hline Total & 32 & 33 & 49 \\
\hline
\end{tabular}

TABLE I

Number of mosquitoes from two species and its genders captured per day by two distinct methods, the BG-Sentinel (BGS-Trap) and the backpack aspirator in a field test conducted at Tubiacanga, Rio de Janeiro

\begin{tabular}{|c|c|c|c|c|c|c|c|c|}
\hline \multirow{2}{*}{$\begin{array}{l}\text { Days after } \\
\text { release }\end{array}$} & \multicolumn{2}{|c|}{ o Culex quinquefasciatus } & \multicolumn{2}{|c|}{ +Cx. quinquefasciatus } & \multicolumn{2}{|c|}{${ }^{\top}$ Aedes aegypti } & \multicolumn{2}{|c|}{ +Ae. aegypti } \\
\hline & BGS-Trap & Aspiration & BGS-Trap & Aspiration & BGS-Trap & Aspiration & BGS-Trap & Aspiration \\
\hline 1 & 87 & 183 & 75 & 236 & 15 & 12 & 7 & 6 \\
\hline 2 & 195 & 106 & 183 & 100 & 12 & 1 & 21 & 1 \\
\hline 3 & 110 & 223 & 69 & 175 & 7 & 1 & 4 & 2 \\
\hline 4 & 20 & 63 & 21 & 62 & 4 & 3 & 7 & 2 \\
\hline 7 & 152 & 52 & 208 & 36 & 10 & 0 & 27 & 6 \\
\hline 8 & 112 & 93 & 69 & 67 & 14 & 3 & 23 & 3 \\
\hline 9 & 26 & 102 & 28 & 67 & 0 & 4 & 5 & 2 \\
\hline 10 & 128 & 108 & 60 & 70 & 7 & 0 & 13 & 0 \\
\hline Total & 830 & 930 & 713 & 813 & 69 & 24 & 107 & 22 \\
\hline
\end{tabular}


A) Male Aedes aegypti

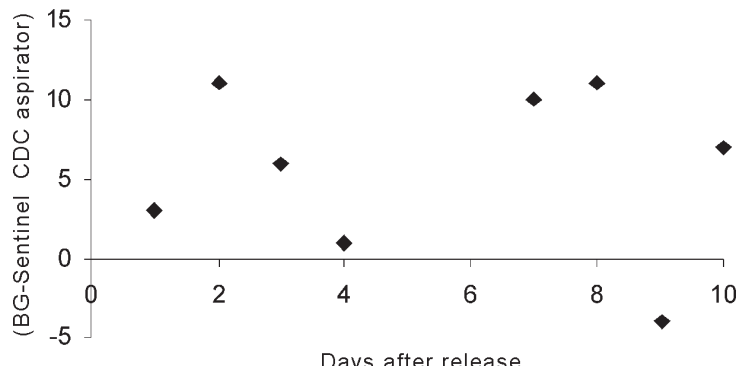

Days after release

B) Female Aedes aegypti

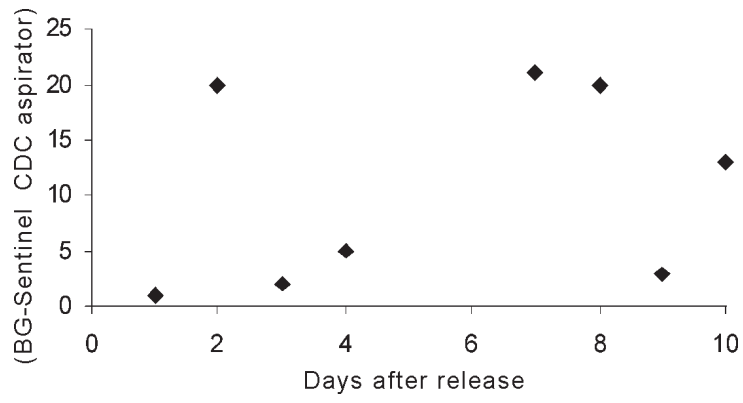

C) Male Culex quinquefasciatus

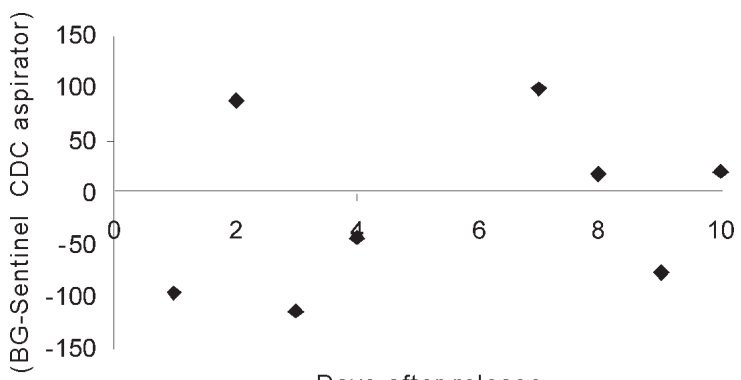

Days after release

D) Female Culex quinquefasciatus

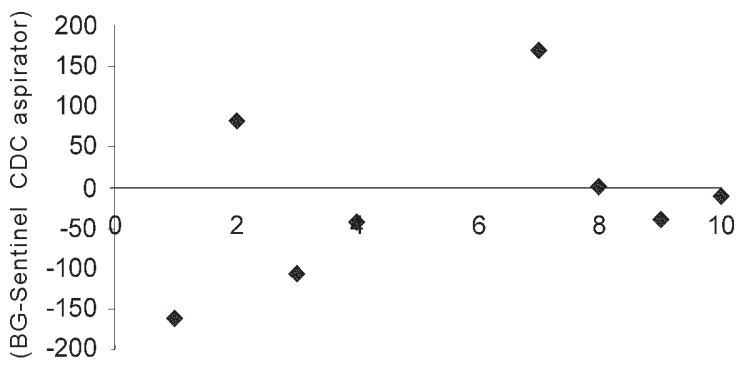

Days after release

Fig. 2: comparison between the BG-Sentinel (BGS-Trap) and the $\mathrm{CDC}$-aspirator to capture adult mosquitoes. Points above the " $x$ " axis means higher efficiency of the BGS-Trap.

seeking females were captured. Finally, in Cohort 3, 44 $(89.8 \%)$ host-seeking females were captured, 4 (8.2\%) females had ovarian in intermediary stages, and just one (2\%) had ovarian in stage $\mathrm{V}$. These results strongly suggest that BGS-Traps capture more host-seeking females in detriment of females in other physiological and behavioral stage.

\section{DISCUSSION}

This report evaluated the efficiency of a new mosquito trap, the BGS-Trap. This trap was originally developed to capture adult Ae. aegypti as a monitoring tool and as an attempt to reduce adult mosquito density, what would finally decrease dengue transmission.

In the present study, BGS-Trap captured more Ae. aegypti in comparison with the backpack aspirator, a widely used method for field collection. Besides, the majority of females captured by BGS-Trap were Ae. aegypti in the initial stage of ovarian development, i.e. host-seeking for blood-feeding. It also captured a high number of males, even with a dispenser which releases a defined combination of substances that are found on human skin, stimulating host-seek female behavior.

Collecting male mosquitoes by the backpack aspirator is common because they are likely to be resting or active

A) Cohort $1=$ Old age, parous, and blood-fed females

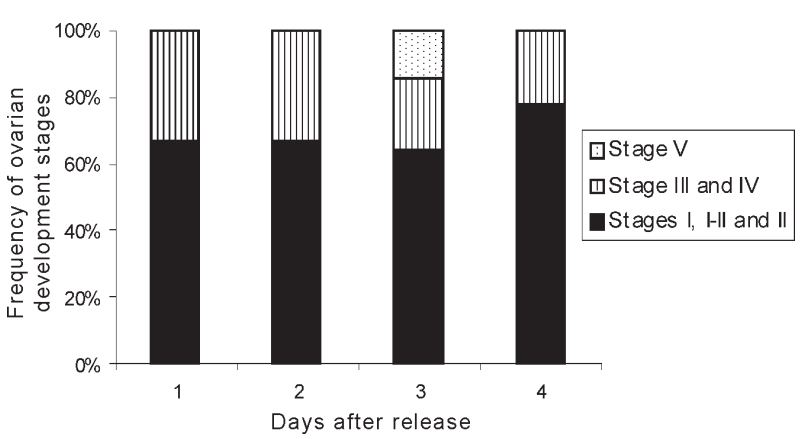

B) Cohort 2 = New age, nulliparous, and blood-fed females

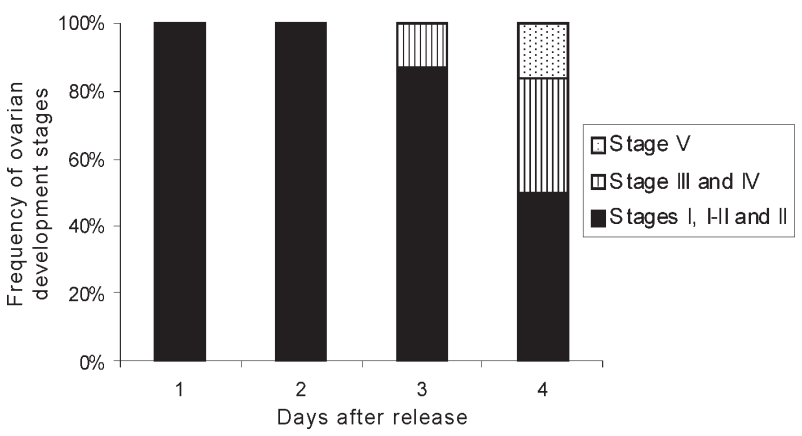

C) Cohort 3 = New age, nulliparous, and starved females

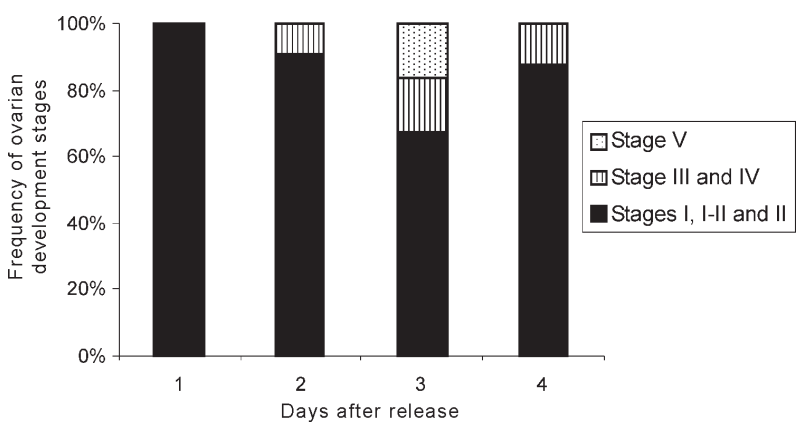

Fig. 3: frequency of ovarian development stages of Aedes aegypti females captured in BG Sentinel-Traps installed in Tubiacanga district, Rio de Janeiro city, Brazil. 
indoors. However, the male mosquitoes being caught by BGS-Trap which uses host odours as attractants is surprisingly. It is well known that males occur around human hosts where they can intercept and mate with females seeking blood meals (Hartberg 1971). It is likely that the trap devices used in this study have visual and olfactory stimuli that attract males towards humans. In spite of being designed and developed to capture Ae. aegypti mosquitoes, $C x$. quinquefasciatus males and females were collected in high numbers, particularly when compared with the former species. However, it is still not clear yet if one of these two caught species is more attracted to the BGS-Trap than the other. We also observed that the backpack aspirators also captured more $C x$. quinquefasciatus than Ae. aegypti. Second, the former species seems to be naturally more abundant in the area than the latter (RMF, unpublished data). Possibly, BGS-Traps can also be used to monitor $C x$. quinquefasciatus, a known vector of human filariasis in Brazil (Brito et al. 1997).

Even capturing a higher number of $C x$. quinquefasciatus mosquitoes, the BGS-Trap proved to be a reliable and more specific method for collecting Ae. aegypti. First, because the BGS-Trap caught almost five times more Ae. aegypti females and almost three times more Ae. aegypti males than backpack aspirator. Moreover, in daily capture, BGS-Trap captured more Ae. aegypti females in all days. These results show a constant, standardized, and reliable tool as potential for detecting and monitoring Ae. aegypti populations in urban areas. Unfortunately, the number of mosquitoes collected by a backpack aspirator is extremely dependent of operator's motivation and skill. The number of Ae. aegypti mosquitoes caught in BGS-Traps was not significantly different regarding the place where the trap was installed.

BGS-Traps captured extremely more Ae. aegypti females in the initial stages of ovarian development, i.e. host-seeking females. Probably, females captured in the intermediary stages of ovarian development (Stages IIIIV) were seeking for a second blood meal before completing the gonotrophic cycle. About $45 \%$ of Ae. aegypti females took multiple blood meals during a single gonotrophic cycle in field experiments conducted in Thailand (Scott et al. 1993).

The results presented in this report reinforce the possibility of using BGS-Traps to help on understanding dengue transmission in an area. Briefly, BGS-Traps can be used to monitor virus circulation since captured females are alive and virus may be isolated from them, as well as to estimate dispersal, survival, and parity rate of mosquito populations.

\section{ACKNOWLEDGMENTS}

To Kléber Soares, Renato Carvalho, Gláuber Rocha, Marcelo Celestino dos Santos, Mauro Menezes Muniz, and Reginaldo Rego for field assistance.

\section{REFERENCES}

Brito AC, Williams P, Fontes G, Rocha EMM 1997. A comparison of two brazilian populations of Culex quinquefasciatus (Say 1823) from endemic and non-endemic areas to infections with Wuchereria bancrofti (Cobbold 1877). Mem Inst
Oswaldo Cruz 92: 33-36.

Christophers SR 1911. The development of the egg follicle in anophelines. Paludism 2: 73-89.

Clark GG, Seda H, Gubler DJ 1994. Use of the "CDC backpack aspirator" for surveillance of Aedes aegypti in San Juan, Puerto Rico. J Am Mosq Control Assoc 10: 119-124.

Consoli RAGB, Lourenço-de-Oliveira R 1994. Principais Mosquitos de Importância Sanitária do Brasil, Fiocruz, Rio de Janeiro, $255 \mathrm{pp}$.

Detinova TS 1945. Determination of the physiological age of female Anopheles from the changes of the tracheal system of the ovaries. Med Parazitol Parazitarn Bolezni 14: 45-49.

Detinova TS 1949. Physiological changes of ovaries in females of Anopheles maculipennis. Med Parazitol Parazitarn Bolezni 18: 410-410.

Focks DA 2003. A Review of Entomological Sampling Methods and Indicators for Dengue Vectors. Special Program for Research and Training in Tropical Diseases (TDR), UNICEF, UNDP, World Bank, World Health Organization. http://www.who.int/tdr/publications/publications/pdf/ dengue_vectors.pdf

Hartberg WK 1971. Observations on the mating behavior of Aedes aegypti in nature. Bull WHO 45: 847-850.

Jensen T, Willis OR, Fukuda T, Barnard DR 1994. Comparison of bi-directional Fay, omnidirectional, CDC, and duplex cone traps for sampling adults Aedes albopictus and Aedes aegypti in north Florida. J Am Mosq Control Assoc 10: 74-78.

Kröckel U, Rose A, Eiras A, Geier M 2006. New tools for surveillance of adult Aedes aegypti: comparison of trap catches with human landing collection in an urban environment. J Am Mosq Control Assoc in press.

Lima-Camara TN, Honório NA, Lourenço-de-Oliveira R 2006. Freqüência e distribuição espacial de Aedes aegypti e Aedes albopictus (Diptera: Culicidae) em distintos ambientes no Rio de Janeiro. Cad Saúde Públ in press.

Maciel-de-Freitas R, Brocki-Neto RW, Gonçalves JM, Codeço CT, Lourenço-de-Oliveira R 2006. Movement of dengue vectors between the human modified environment and an urban forest in Rio de Janeiro. J Med Entomol in press.

Ordonez-Gonzalez JG, Mercado-Hernandez R, Florez-Suarez AE, Fernandez-Salas I 2001. The use of sticky ovitraps to estimate dispersal of Aedes aegypti in Northeastern Mexico. J Am Mosq Control Assoc 17: 93-97.

Rutledge LC, Ward RA, Gould DJ 1964. Studies on the feeding response of mosquitoes to nutritive solutions in a new membrane feeder. Mosq News 24: 407-419.

Scott TW, Chow E, Strickman D, Kittayapong P, Wirtz RA, Lorenz LH, Edman JD 1993. Blood-feeding patterns of Aedes aegypti (Diptera:Culicidae) collected in a rural Thai village. J Med Entomol 30: 922-927.

Service MW 1993. Mosquito Ecology - Field Sampling Methods, Chapman \& Hall, London, 988 pp.

Sokal RR, Rohlf FJ 2001. Biometry, WH Freeman and Company, New York, 887 pp.

Tsuda Y, Takagi M, Wang S, Wang ZM, Tang L 2001. Movement of Aedes aegypti (Diptera: Culicidae) released in a small isolated village on Hainan Island, China. J Med Entomol 38: 93-98. 